\title{
Market Access Analysis of Biologics and Small-Molecule Inhibitors for Inflammatory Bowel Disease Among US Health Insurance Policies
}

\author{
Parambir S. Dulai ${ }^{1} \cdot$ Mark T. Osterman $^{2} \cdot$ Karen Lasch $^{3} \cdot$ Charlie Cao $^{3} \cdot$ Faisal Riaz ${ }^{3} \cdot$ William J. Sandborn $^{1}$
}

Received: 27 April 2018 / Accepted: 18 March 2019 / Published online: 28 March 2019

(c) The Author(s) 2019

\begin{abstract}
Background and Aims Treatment pathways for ulcerative colitis (UC) and Crohn's disease (CD) are shifting to a more individualized, risk-stratified approach. The perception is that insurance policies may not have implemented this paradigm shift, particularly regarding access to newer agents. We evaluated patient access to advanced therapies by analyzing policy information from the Managed Markets Insight and Technology database.

Methods Coverage status as of December 2018 for all US lives was queried for adalimumab, infliximab, infliximab-dyyb, tofacitinib, ustekinumab, and vedolizumab by indication (UC and/or CD) and medical or pharmacy coverage benefit. Coverage status was classified by the number of biologic steps before access to specified drug as "No Biologic," "1 Prior Biologic," "2+ Prior Biologics," "Not Covered." Unknown lives were excluded from the analyses.

Results Coverage analysis was available for approximately 302 million lives under each medical and pharmacy benefit. Our analysis indicates that approximately half of covered lives had access to all agents (except tofacitinib) as first-line therapy; two-thirds had access after one biologic exposure. Among newer agents, vedolizumab had the widest coverage. For indications of UC and CD, $81 \%$ of known lives had access to vedolizumab with no prior biologic exposure required ("No Biologic"), 95\% after "No Biologic" + "1 prior Biologic." Geographic variations were identified for coverage patterns.

Conclusions This US-based healthcare policy analysis points to an increased access to advanced therapies for UC and CD. An individualized, risk-stratified treatment approach integrating advanced therapies, including those recently approved, into treatment pathways for UC and CD is feasible.
\end{abstract}

Keywords Ulcerative colitis · Crohn's disease $\cdot$ Health insurance coverage $\cdot$ Vedolizumab $\cdot$ Ustekinumab $\cdot$ Tofacitinib

Electronic supplementary material The online version of this article (https://doi.org/10.1007/s10620-019-05594-7) contains supplementary material, which is available to authorized users.

Parambir S. Dulai

pdulai@ucsd.edu

Mark T. Osterman

mark.osterman@uphs.upenn.edu

Karen Lasch

karen.lasch@takeda.com

Charlie Cao

charlie.cao@takeda.com

Faisal Riaz

faisal.riaz@takeda.com

\section{Introduction}

The goal of inflammatory bowel disease (IBD) management is to induce and maintain corticosteroid-free clinical and endoscopic remission, while minimizing disease- and treatment-related adverse events [1-3]. To achieve this goal, treatment strategies have traditionally followed a step-up

William J. Sandborn

wsandborn@ucsd.edu

1 Division of Gastroenterology, University of California San Diego, 9500 Gilman Dr, La Jolla, CA 92093, USA

2 Division of Gastroenterology, University of Pennsylvania, 51 North 39th St, Philadelphia, PA 19104, USA

3 US Medical Office, Takeda Pharmaceuticals, U.S.A., Inc, One Takeda Parkway, Deerfield, IL 60015, USA 
approach, in which patients are required to fail multiple immunosuppressive agents before starting a biologic agent $[1,3]$. However, this step-up approach may not represent the optimal strategy, given that using agents with low efficacy for a prolonged duration allows inflammation to continue and tissue damage to occur [4]. Updated treatment guidelines supported by the American Gastroenterological Association (AGA) have evolved toward an individualized, riskstratified therapeutic approach, with the early integration of biologic therapy for high-risk patients [5-7]. As a result, biologic drugs are becoming the standard of care given their favorable efficacy and safety profiles compared with immunomodulators [8].

The ability to use biologic therapies in clinical practice, however, is in part dictated by insurance company policies and their specification of preferred agents. A survey published in 2017 of the 125 largest US insurance companies found that over $90 \%$ of policies were not compliant with the AGA clinical pathways for ulcerative colitis (UC) and Crohn's disease (CD) [9]. Thus, healthcare coverage and access to therapy may not be consistent with treatment guidelines and recommendations. The gaps between coverage and optimal (or guideline-recommended) treatment may be more relevant for recently approved biologic therapies, as well as evolving small-molecule inhibitor therapies, and these gaps may affect the speed at which these therapies are adopted, regardless of their clinical benefit.

In recent years, the treatment landscape for IBD in the USA has seen several approvals of new agents beyond tumor necrosis factor (TNF) antagonists such as infliximab, infliximab biosimilars, and adalimumab. In 2014, the FDA approved vedolizumab, a humanized, gut-selective, monoclonal antibody that inhibits the migration of $\alpha_{4} \beta_{7^{-}}$ expressing T lymphocytes into the gut tissue, for use in adult patients with moderately to severely active UC and CD [10, 11]. Subsequently, ustekinumab, a human monoclonal antibody against human interleukin 12 and interleukin 23 used for the treatment of moderately to severely active $\mathrm{CD}$, and tofacitinib, a small-molecule inhibitor of the JAK1 and JAK3 pathways for the treatment of moderately to severely active UC, were approved in 2016 and 2018, respectively [12, 13]. Each of these treatments has distinct clinical features, such as the potential safety advantages of being gut-selective for vedolizumab [14], or the convenience of oral administration for tofacitinib, that may make them attractive options, particularly for certain populations. Yet, it is unclear whether insurance coverage for these novel treatments as a first- or second-line agent is routinely approved in clinical practice. This uncertainty could have an impact on their appropriate integration into current treatment pathways, which has not been evaluated to date.

It would therefore be informative to more comprehensively revisit the intention of the prior report [9] and reflect on whether there has been any progress in bringing together the recommendations from authoritative guidelines and the actual possibilities based on real-world access, especially now, with even more agents in the treatment armamentarium. In this study, we evaluated patient access to advanced therapies overall, and to novel agents in particular, by analyzing policy information from the Managed Markets Insight and Technology (MMIT) LLC database.

\section{Materials and Methods}

\section{Database Search}

Data were extracted from the MMIT database, database as of December 18, 2018, and reported as number of lives covered under commercial, Medicare, state and managed Medicaid, and Health Exchanges. MMIT was chosen as a resource because of its extensive coverage of medical benefits and real-time assessments of approximately 3185 medical and 6653 pharmacy benefit plans with nightly updates and rapid investigation into policy changes. The estimated proportions of plans represented in the MMIT database are approximately $82 \%$ local (defined as plans operating in 1 state), $14 \%$ regional (defined as plans operating in 2-30 states), and $4 \%$ national (defined as plans operating in $>30$ states). Of note, the percentage of plans within each category is not indicative of the number of lives contributed by each plan type. For this type of study, formal consent is not required.

\section{Drug Access Calculation}

Using MMIT's Analytics 3 Web-based tool, coverage status as of December 18, 2018, for all US lives was queried for adalimumab, infliximab, infliximab-dyyb, tofacitinib, ustekinumab, and vedolizumab by indication (UC and/or CD) and medical or pharmacy coverage benefit. To understand how coverage patterns varied among treatments administered by intravenous (IV) infusions, access was compared between TNF antagonist agents, including infliximab and an infliximab biosimilar (infliximab-dyyb), vedolizumab, and ustekinumab (IV). These intravenous agents were covered primarily under the medical benefit, and therefore "medical coverage/lives" were used for access calculations. Adalimumab (subcutaneous [SC] injection), tofacitinib (oral), and ustekinumab (SC injection) are covered under pharmacy benefit; therefore, "pharmacy coverage/lives" were used for access calculations.

MMIT collects source documentation and determines coverage by digital collection methods (including digital stream from payers and pharmacy benefit managers, artificial intelligence to monitor payer-searchable sites, and published formulary documents) and manual collection 
when needed. Through the manual process, MMIT collects formulary data via pdf and phone outreach from the payers when coverage cannot be confirmed via digital collection methods.

Coverage status for all drugs was assessed by MMIT's team based on publicly available medical policies, prior authorization (PA), and pharmacy drug list documents. If these policies and/or documents are not publicly available for a plan, the coverage is classified as "Unknown," "Covered (PA details unknown)," or "Covered (step therapy [ST] Details Unknown)." Lives with unknown coverage status arise from small regional plans that do not publicly publish detailed medical policies or from plans where prior authorization is specified on their formulary but coverage restriction details are unknown. When coverage status is known, all known lives were classified based on the number of prior biologic exposures before access to the specified drug as "No Biologic," "1 prior Biologic," "2+ prior Biologics," and "Not Covered" (Fig. 1). The number of prior biologic exposures represents individual exposures of any biologic within the same or different class. An exposure could be a trial of, failure of, contraindication to, or other interaction with a biologic, as defined by the insurance payer. A biologic therapy could include infliximab, adalimumab, ustekinumab, vedolizumab, golimumab, certolizumab, or biosimilar infliximab, but the specific required biologic exposure is defined by the payer in the medical policy.

\section{Regional Access Variations}

To assess regional variations, coverage status for vedolizumab (UC and CD indications combined), ustekinumab (CD only), and tofacitinib (UC only) was filtered by "No Biologic" and "No Biologic" + "1 prior Biologic" to identify the total number of covered lives by state. The numbers of lives were calculated at a state level using a weighted average and then adjusted for the census by dividing the number of covered lives by the July 2017 state census population. MMIT data represent $100 \%$ of the insured state populations. The methodology used takes into account census data for each state (to assign the total lives in each state), as well as the national uninsured rate (used to adjust each state's population) and the AIS Health survey, which is the primary source of medical lives information.

\section{Results}

\section{Overall Coverage}

In total, $301,748,186$ and $302,057,543$ unique lives were captured in the MMIT database under the medical and pharmacy benefit, respectively.

Overall, over $89 \%$ of lives required no or 1 prior biologic use ("No Biologic" + "1 prior Biologic") before access to any of the drugs for the indications of $\mathrm{UC}$ or $\mathrm{CD}$, with the
Fig. 1 Methodology for drug market access determination. ${ }^{a}$ Unknown and step therapy (ST Unknown) Status: Primarily for smaller regional plans $(70 \%$ of unknown lives are from plans with <300,000 lives). Managed Medicaid plans are most prominent among these unknown statuses, followed by commercial PPO plans. Typically, these types of smaller regional plans do not publicly publish detailed medical policies. ${ }^{\text {b}}$ Prior Authorization (PA Unknown) Status: Plans where a PA is specified on their formulary; however, the details of the PA are not available and therefore cannot be assessed. PA restrictions are in place, but the details of those restrictions are unknown. PPO, preferred provider organization

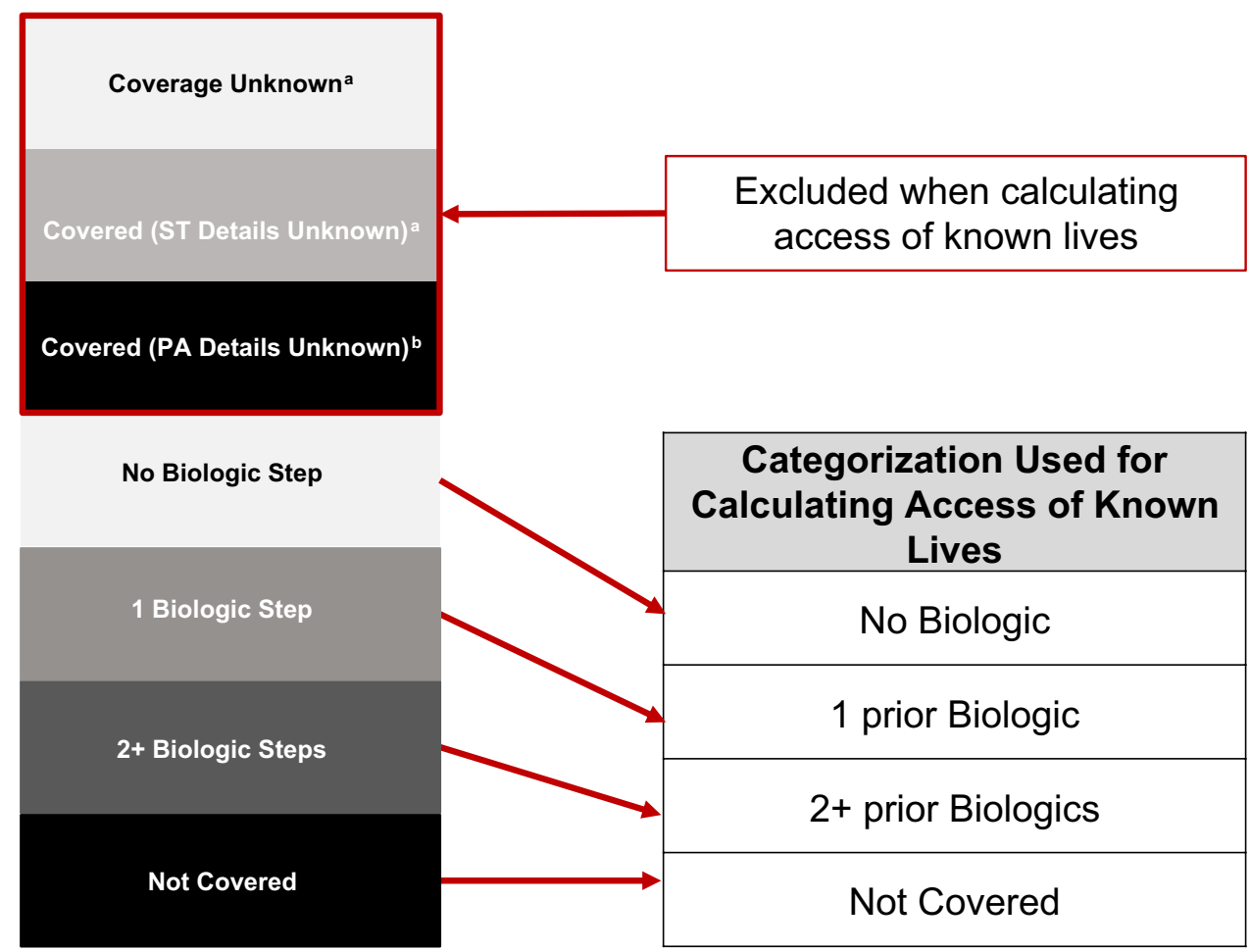


exception of tofacitinib (UC) and ustekinumab SC (CD), for which access was $68 \%$ and $71 \%$, respectively (Fig. 2).

The products with wider coverage were infliximab and adalimumab. Access to infliximab and its biosimilar, infliximab-dyyb, under the medical benefit was $95 \%$ and $89.8 \%$ ("No Biologic" + "1 prior Biologic") for the indication of UC and $95 \%$ and $90 \%$ for the indication of CD, respectively. Adalimumab access under pharmacy benefit was $99 \%$ ("No Biologic" + "1 prior Biologic") for the indications of UC and CD (Fig. 2).

\section{Coverage of Novel Agents}

Among newer agents, vedolizumab appeared to have the widest coverage. For the indications of UC and CD, $81 \%$ of known lives had access to vedolizumab with no prior biologic exposure required ("No Biologic"), 95\% after "No Biologic" + "1 prior Biologic," $4 \%$ after " $2+$ prior Biologics," and $2 \%$ of lives had no coverage (Fig. 2). For the indication of UC, $43 \%$ of known lives had access to tofacitinib with no prior biologic exposure required ("No Biologic"), $68 \%$ after "No Biologic" + "1 prior Biologic," $13 \%$ after "2+ prior Biologics," and 19\% of lives had no coverage. Ustekinumab coverage for the indication of $\mathrm{CD}$ varied based on the route of administration; ustekinumab IV was covered under medical benefit and ustekinumab SC under pharmacy benefit. Rate of coverage for ustekinumab SC was lower than for ustekinumab IV; $86 \%$ and $49 \%$ of known lives had access to ustekinumab IV and SC, respectively, without prior biologic exposure, and $94 \%$ and $71 \%$ after "No Biologic" + "1 prior Biologic."

For the UC indication, $\sim 7 \%$ and $\sim 16 \%$ of lives had unknown coverage under medical and pharmacy benefit, respectively, and for the $\mathrm{CD}$ indication, unknown lives accounted for $\sim 8 \%$ and $\sim 7 \%$ under medical and pharmacy benefit, respectively.

When analyzed by type of insurance, vedolizumab and ustekinumab IV coverage without prior biologic exposure ("No Biologic") was largely similar in commercial and government policies. Under pharmacy benefit, tofacitinib and ustekinumab SC coverage without prior biologic exposure in commercial policies was higher than in government policies. (Supplementary Data Contents 1-3).

\section{Regional Variations in Coverage of Novel Agents}

There was regional variability in access to all drugs evaluated among states. Access rates were lowest for tofacitinib (10-44\%) after "No Biologic" in all states for the UC indication (Fig. 3). Access was highest for vedolizumab after "No Biologic" + "1 prior Biologic," with access rates $>60 \%$ in all states except for North Dakota, Mississippi, and Vermont for the UC and CD indications combined (Fig. 3).

\section{Discussion}

In the present analysis, more than $80 \%$ of covered lives had access to the newer biologics vedolizumab and ustekinumab (IV) without any prior biologic exposure, and only $4 \%$ or fewer lives were required to have an exposure to two or more biologics before having access. This biologicnaïve access is consistent with the approved labeling for these agents, which indicates use after failure of conventional therapy (corticosteroids, immunomodulators) [10, 12]. These observations are in direct contrast to an analysis carried out in 2014 by Yadav et al., who, after analyzing the top 125 insurance policies by market share, concluded that only $11 \%$ and $8 \%$ of the first 50 reviewed policies covered vedolizumab without initial failure of a TNF antagonist in the UC and CD indications, respectively, and that $21 \%$ of policies required the failure of 2 or more TNF antagonists before covering vedolizumab. In the same analysis, $71 \%$ of policies allowed ustekinumab use without prior anti-TNF failure, although only $34 \%$ of the policies had a separate policy for ustekinumab [9]. We conducted our analysis in the same 50 policies, except for one that exited the insurance marketplace in 2015, and found coverage rates for vedolizumab to be higher than those reported in the Yadav et al. study. In fact, our data suggest that, of the 49 policies evaluated, 27 (55\%) covered vedolizumab under "No Biologic" in the UC and CD indications (Supplementary Data Content 4 and 5). In addition, 32 policies $(65 \%)$ covered ustekinumab IV and $8(16 \%)$ covered tofacitinib under "No Biologic" in the CD and UC indications, respectively (Supplementary Data Contents 4 and 5).

Of note, the Yadav analysis did not specify whether the plans included in the analysis were specific to the pharmacy and/or medical benefit. Additionally, the plans in the Yadav analysis only accounted for $\sim 171$ million and $\sim 146$ million lives under medical and pharmacy benefit, respectively, while this analysis captures $\sim 302$ million lives (under each medical and pharmacy benefit) and represents over $\sim 6600$ pharmacy plans and $\sim 3200$ medical plans. Overall, the observed shift in vedolizumab access since the Yadav et al. analysis may be a result of several factors including time-dependent limitations in integrating biologics within insurance policies and approval processes, the evolution of clinical practice data to support the use of vedolizumab early in the biologic treatment cycle of patients, or enhanced understanding of vedolizumab cost-effectiveness.

These data may not reflect individual physician experience and, in fact, may be contrary to their personal understanding of access. It is worth noting the complex and sometimes convoluted steps between writing a prescription and getting a drug to a patient. Benefit investigation, 
a

Medical coverage of known lives ${ }^{a}$ $(301,748,186)$

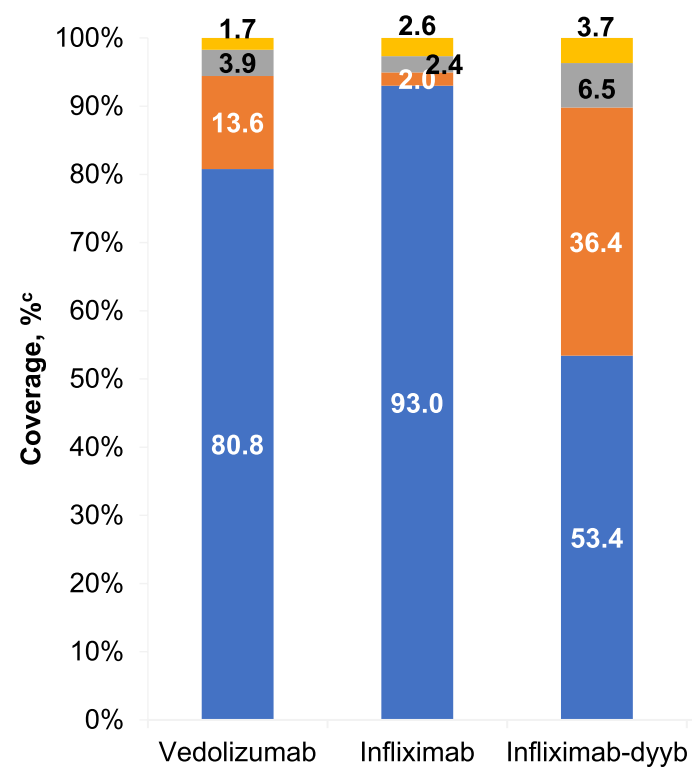

\section{Ulcerative Colitis}

Pharmacy coverage of known lives ${ }^{b}$ $(302,057,543)$

b

\section{Crohn's Disease}

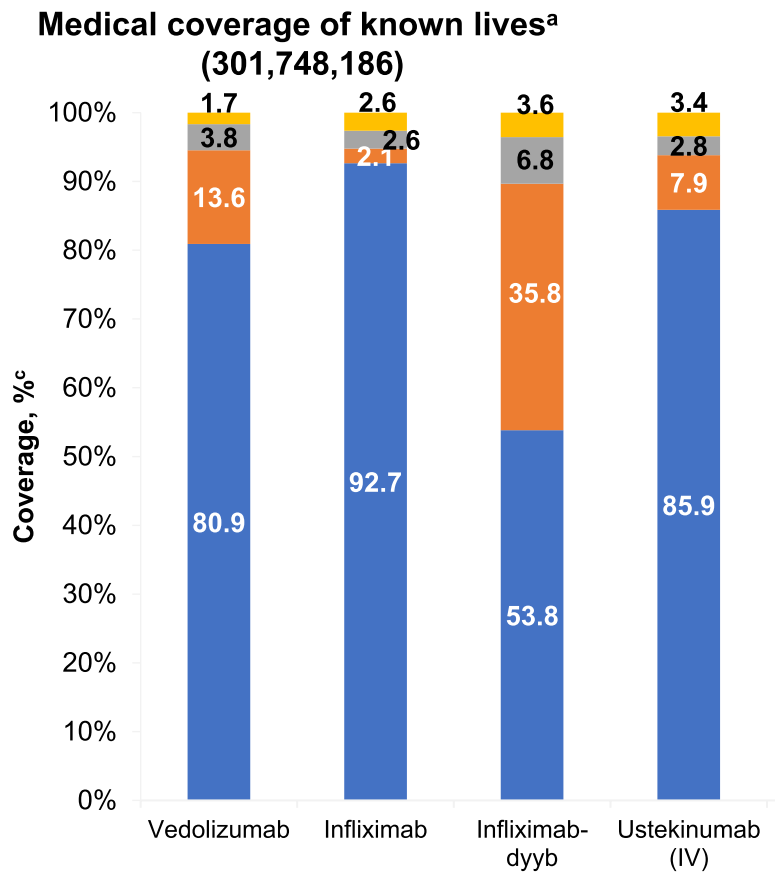

Pharmacy coverage of known lives ${ }^{b}$

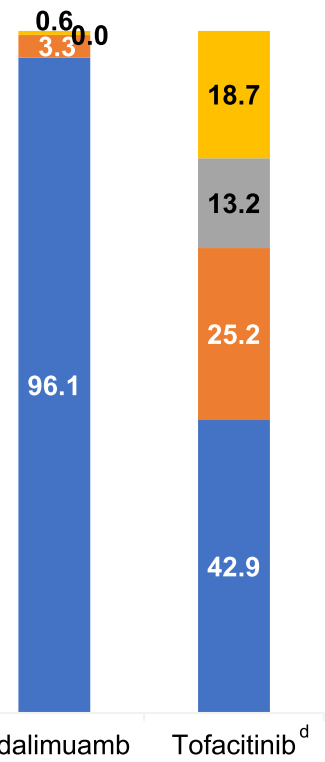

No Biologic

1 Prior Biologic

2+ Prior

Biologics

No Coverage

Adalimuamb Tofacitinib $^{d}$

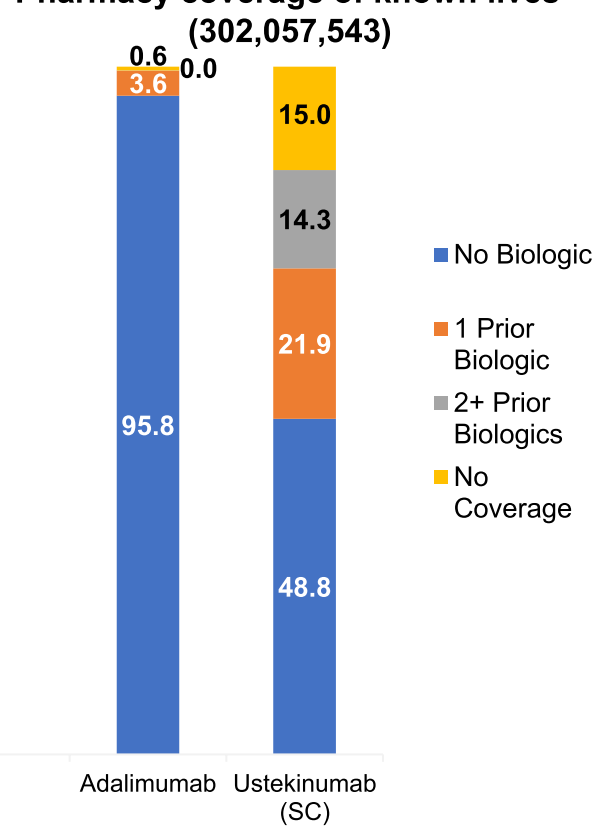

Fig. 2 Drug access under medical coverage for ulcerative colitis and

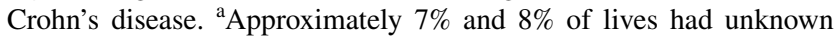
coverage under medical benefit in $\mathrm{UC}$ and $\mathrm{CD}$, respectively. ${ }^{\mathrm{b}} \mathrm{Ap}$ proximately $16 \%$ and $7 \%$ of lives had unknown coverage under pharmacy benefit in $\mathrm{UC}$ and $\mathrm{CD}$, respectively. ${ }^{\mathrm{c}}$ The calculated percentage of covered lives excluded all unknown lives. ${ }^{\mathrm{d}}$ Tofacitinib has a large

percentage of unknown lives $(\sim 25 \%)$, primarily because it was only approved for UC in May 2018. Therefore, this may result in overinflection of the "No Biologic" coverage. CD, Crohn's disease; MMIT, Managed Markets Insight and Technology; UC, ulcerative colitis Source: MMIT, December 18, 2018 


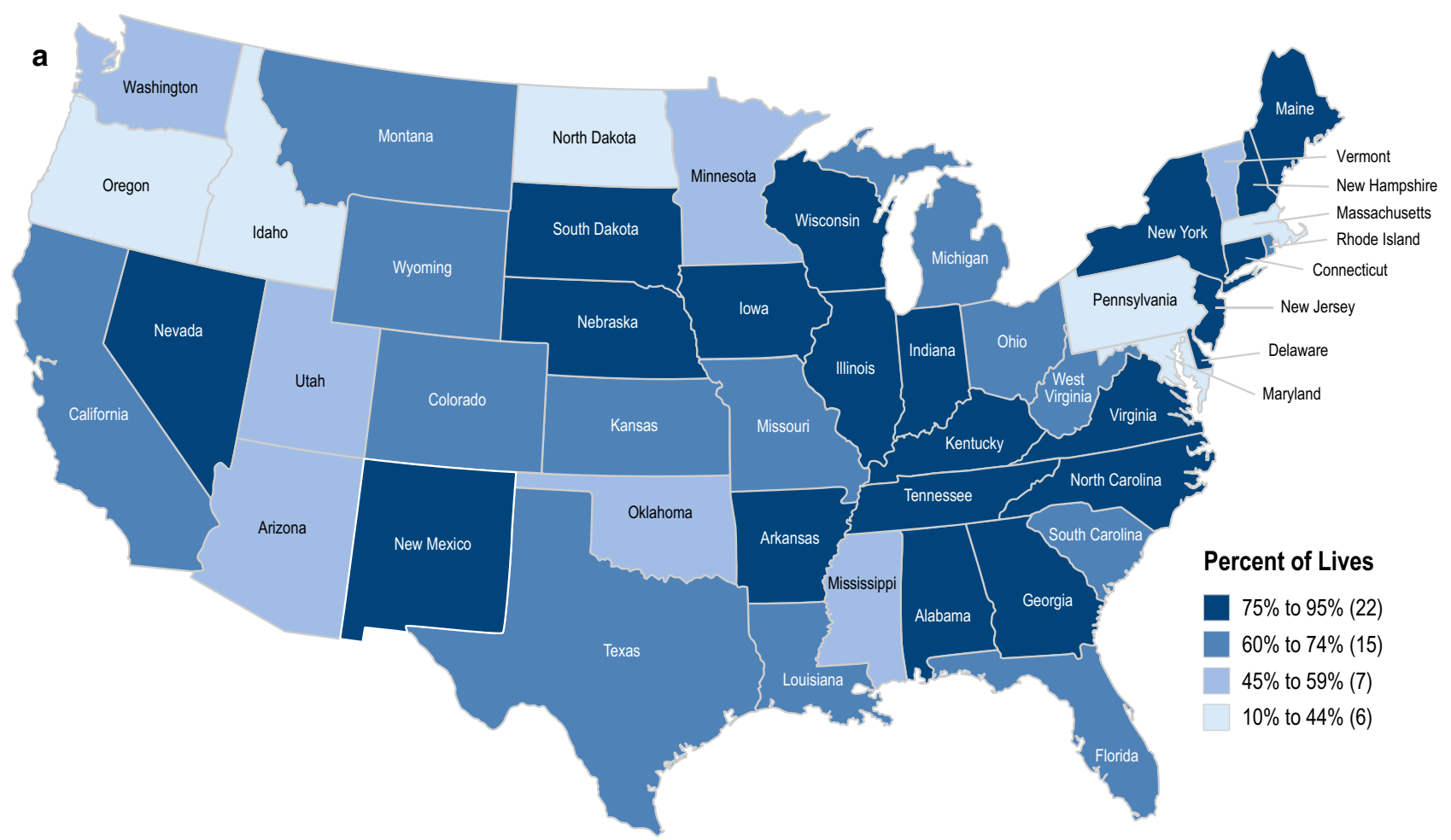

b

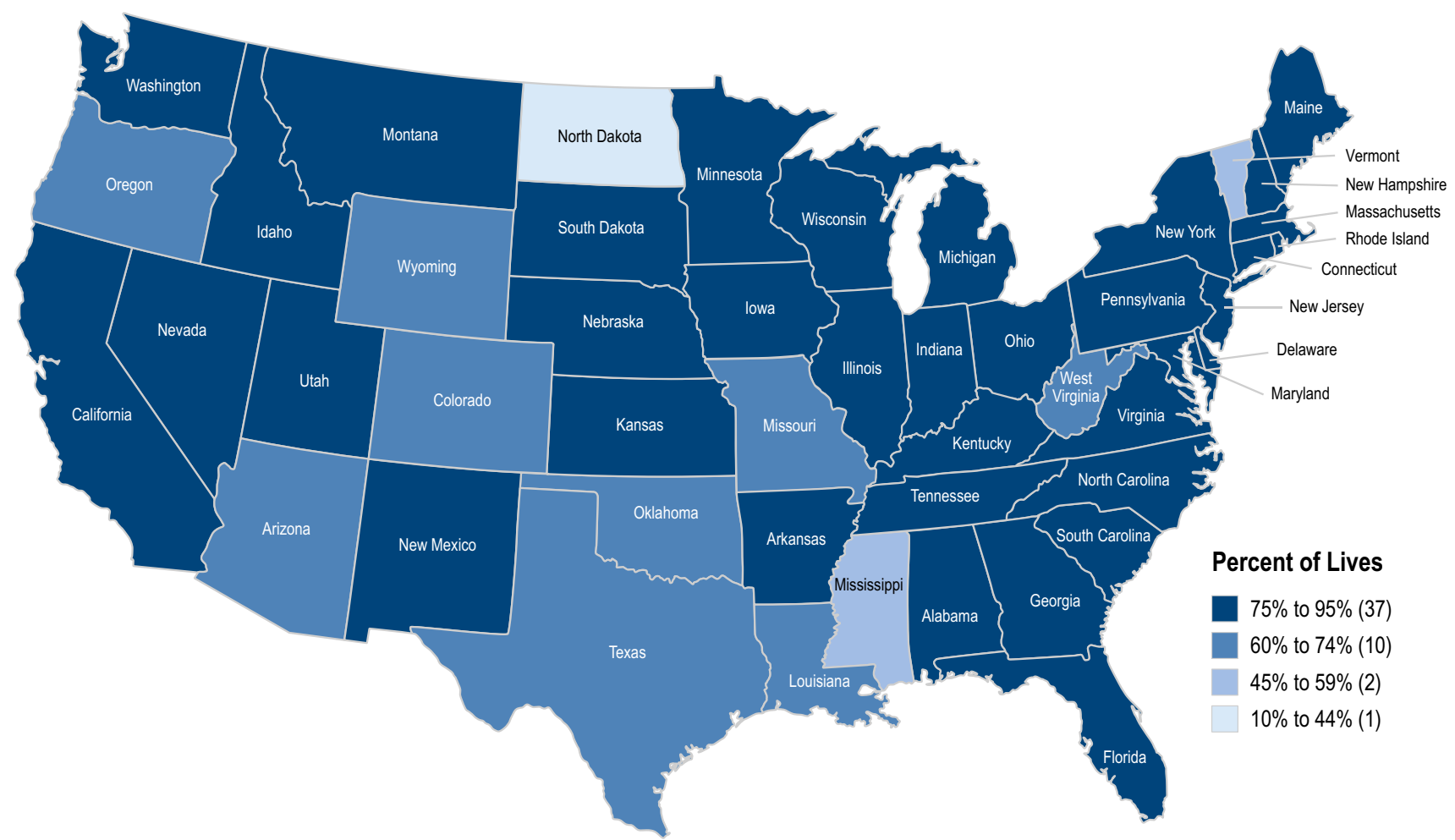

Fig. 3 Access for multiple drugs by state. a Vedolizumab UC and CD (No Biologic). Coverage for Alaska and Hawaii is $73 \%$ and $83 \%$, respectively. Source: MMIT, December 18, 2018. b Vedolizumab UC and $\mathrm{CD}$ (No Biologic +1 prior Biologic). Coverage for Alaska and Hawaii is $73 \%$ and $86 \%$, respectively. c Ustekinumab (IV) CD (No Biologic). Coverage for Alaska and Hawaii is $73 \%$ and $68 \%$, respectively. d Ustekinumab (IV) CD (No Biologic +1 prior Biologic). Coverage for Alaska and Hawaii is $73 \%$ and $71 \%$, respectively. e Ustekinumab (SC) CD (No Biologic). Coverage for Alaska and Hawaii is $26 \%$ and $49 \%$, respectively. f Ustekinumab (SC) CD (No Biologic +1 prior Biologic). Coverage for Alaska and Hawaii is $41 \%$ and $52 \%$, respectively. g Tofacitinib UC (No Biologic). Coverage for Alaska and Hawaii is $28 \%$ and $43 \%$, respectively. $\mathbf{h}$ Tofacitinib UC (No Biologic +1 prior Biologic). Coverage for Alaska and Hawaii is $39 \%$ and $49 \%$, respectively 


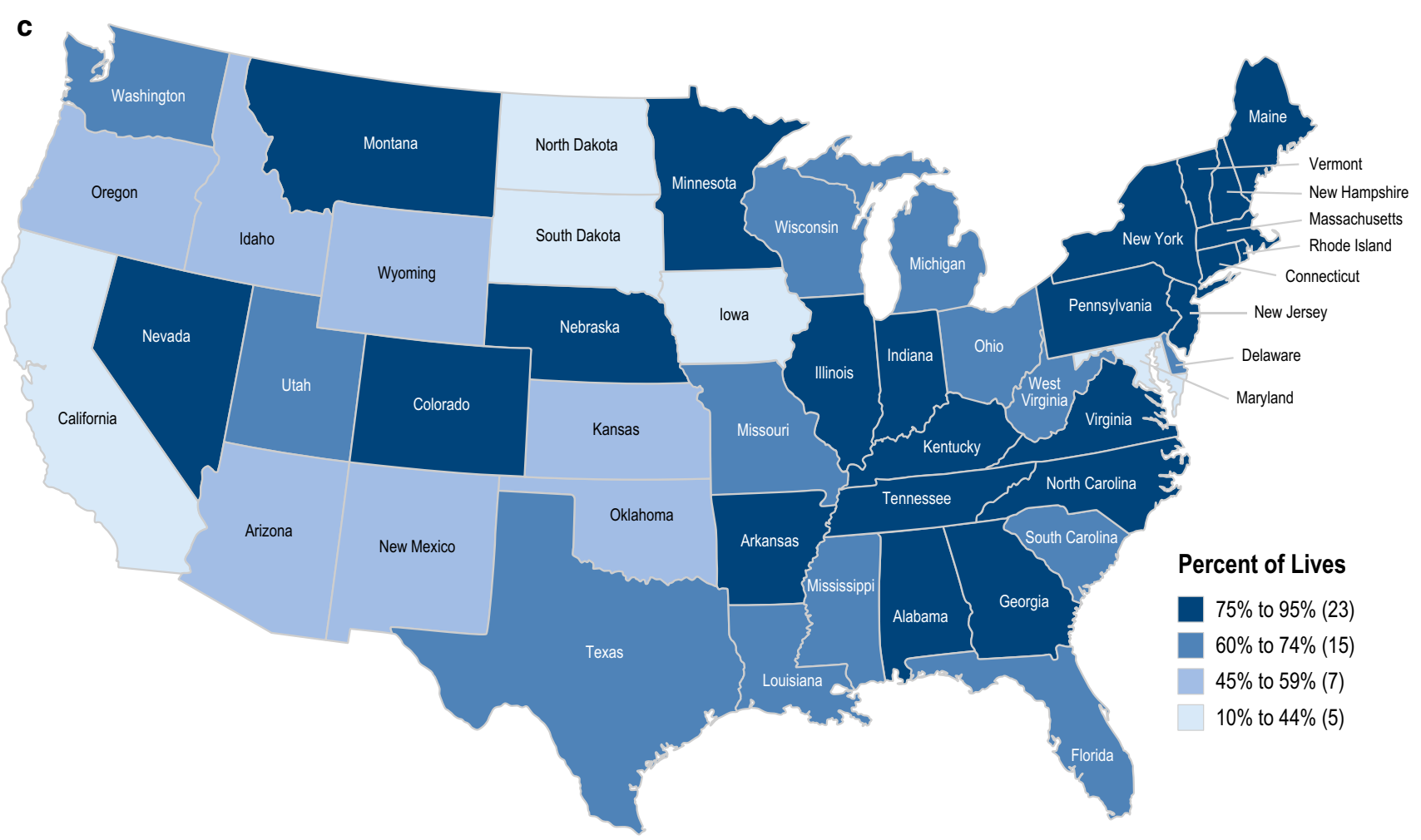

d

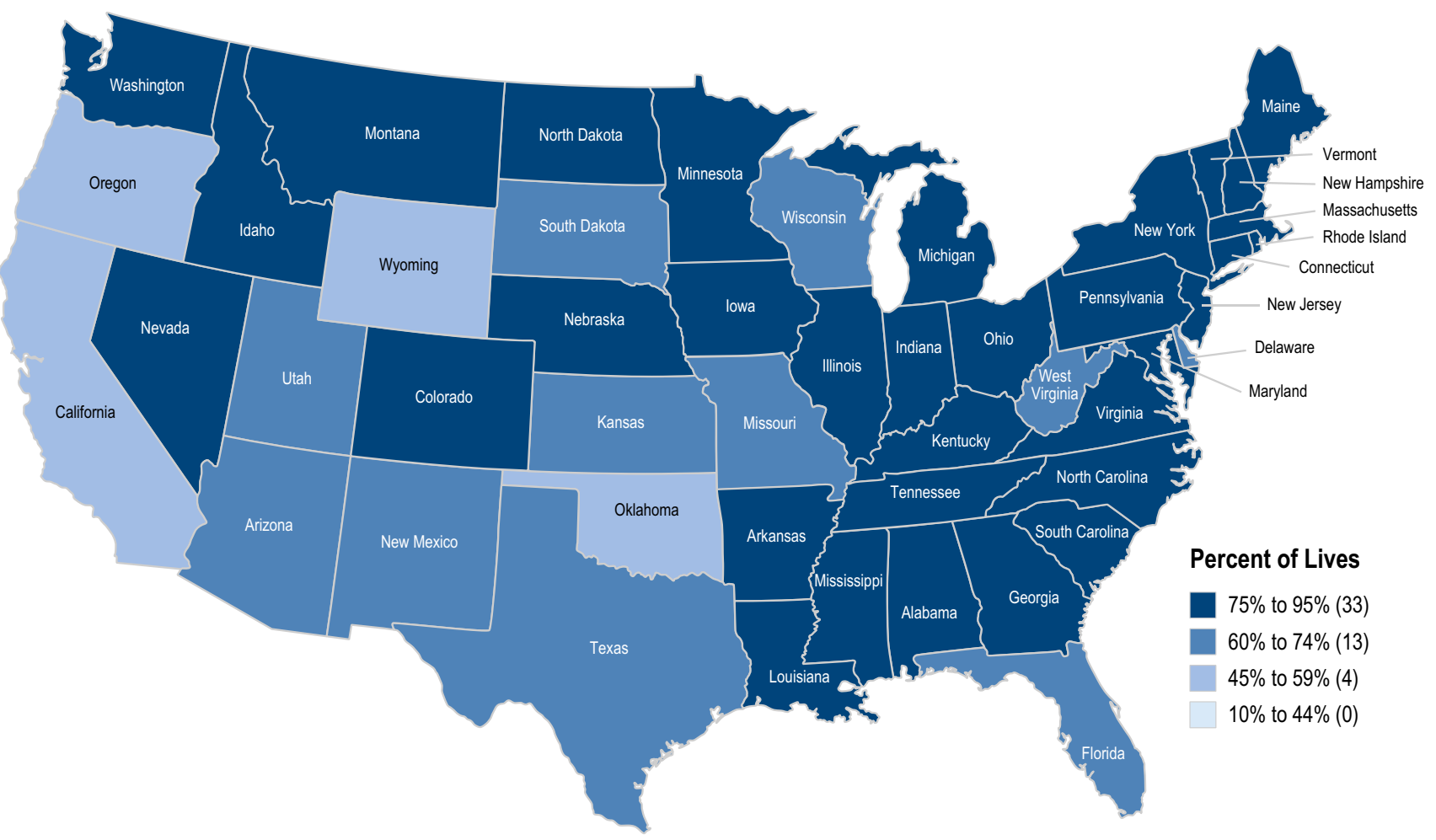

Fig. 3 (continued) 

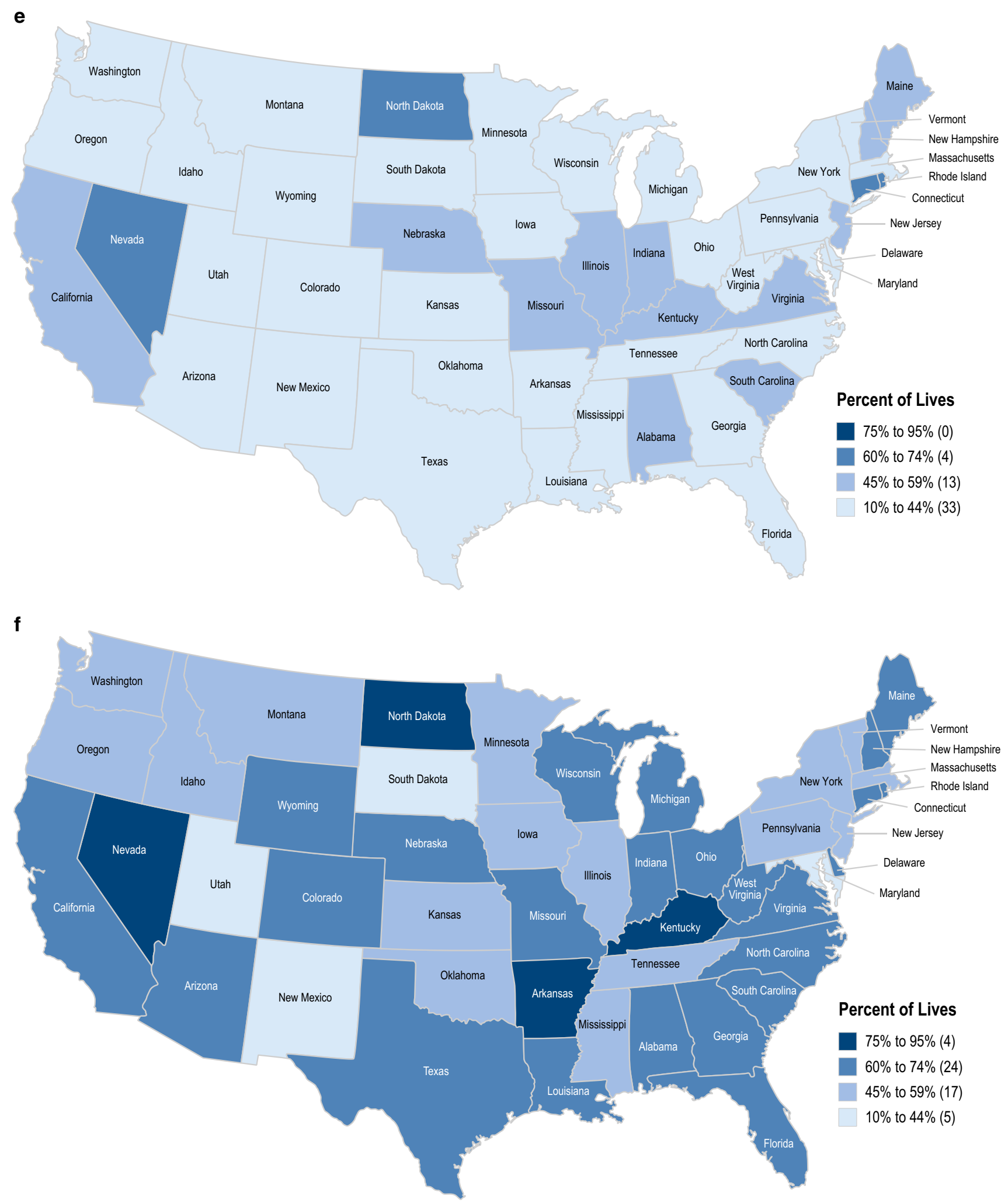

Fig. 3 (continued) 

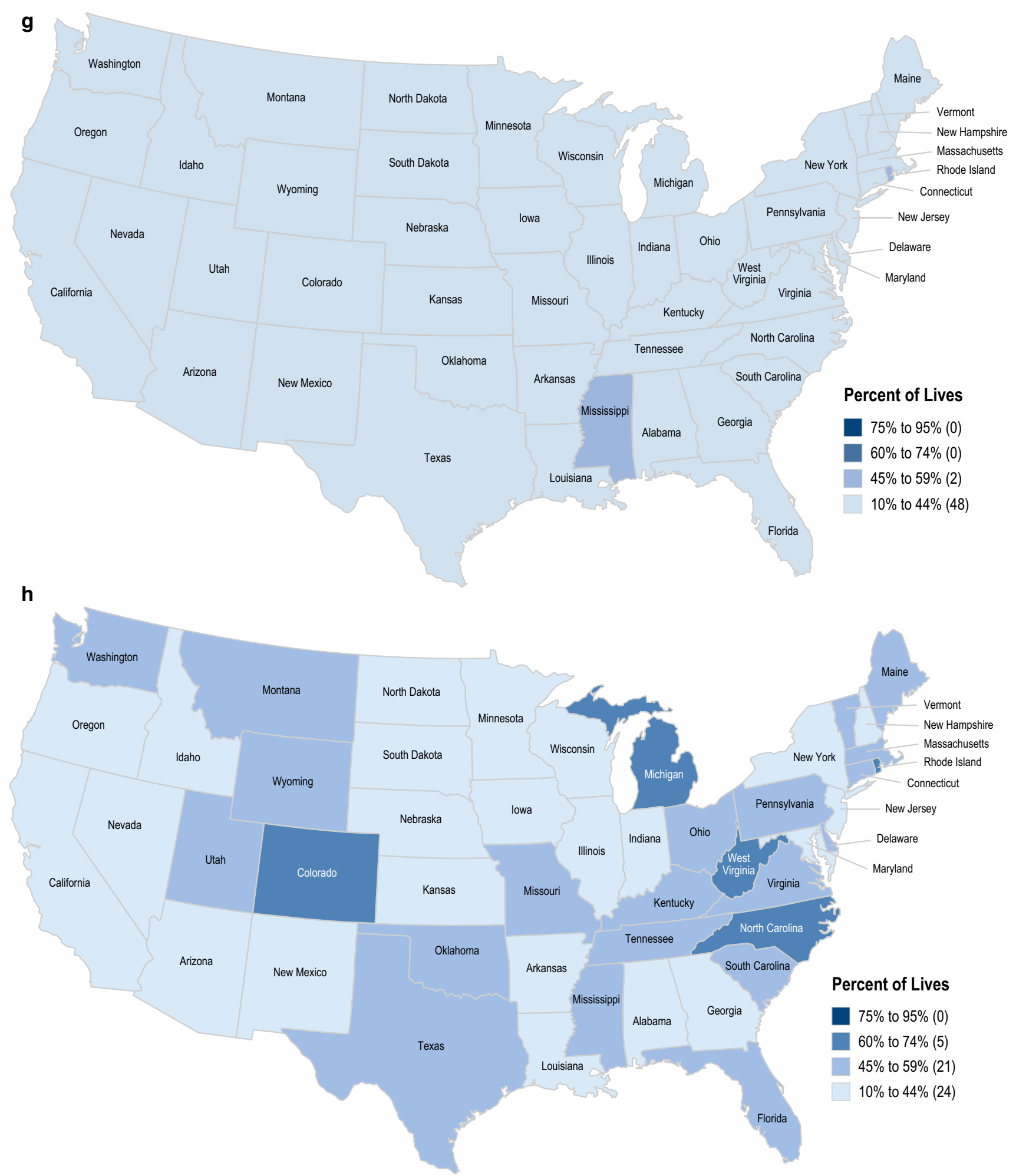

Fig. 3 (continued)

employer plans, deductibles, vaccination, laboratory testing, X-rays, prior authorization paperwork, patient assistance programs, available infusion centers, specialty pharmacy requirements, and patient availability are all potential barriers that are often lumped into "access" issues. In addition, the regional variations show some 
interesting data. Because many insurers rely on local physician demand to dictate policy changes, it may be difficult to determine whether local practices changed access or vice versa.

The ability of providers to use drugs in clinical practice is, in part, dictated by insurance company policies and their preferred agents. In an online survey of US gastroenterologists, nearly half (44\%) of physicians reported experiencing barriers to prescribing biologics for UC. The most commonly cited barrier was patient insurance restrictions (79\%) [15]. Insurance coverage decisions are often based on a mix of unit cost, cost of administration, site of care, market share, manufacturer's rebate, and positioning of an agent or portfolio of agents across multiple autoimmune disease indications. There are limited data and no published head-to-head trials to help inform drug positioning for IBD, although comparative effectiveness and safety studies have been conducted using real-world evidence to help bridge this gap [16, 17]. Results from a 2018 systematic review showed that infliximab and vedolizumab were ranked among the highest first-line agents for clinical remission and mucosal healing in patients with UC [18]. By leveraging their clinical expertise and knowledge of the risk-benefit profile of IBD treatment agents, physicians may be able to educate both patients and payers on the most appropriate agent for each patient. In addition, payers may designate some agents as their preferred agent without considering the relative safety, efficacy, cost, value, or optimal positioning. Introduction of agents to the marketplace with novel mechanisms of action or routes of administration is making traditional treatment paradigms obsolete. Many health plans are now allowing earlier access to more agents. Ideally these access changes will align with the emerging individualized, risk-stratified treatment approach.

Unlike vedolizumab and other infusion therapies that are primarily covered under the medical benefit, self-administered injectables, such as adalimumab or ustekinumab SC, or oral drugs, such as tofacitinib, are primarily covered under the pharmacy benefit. Our results indicate that ustekinumab SC and tofacitinib access was lower than adalimumab. A possible explanation for this result could be the amount of time various treatments have been on the market, coverage for other indications driving larger rebates to health plans, or status as first-in-class treatment per formulation. Alternatively, this result may be explained by the fact that TNF antagonists have been demonstrated to be more effective when used as top-down therapy (vs. step-up therapy) in a randomized controlled trial setting. In fact, earlier use of TNF antagonists was associated with a lower risk of concomitant corticosteroid use, TNF antagonist dose escalation, discontinuation/switch of TNF antagonists, or CD-related surgery [19-21]. As more clinical trial and real-world data on newer agents emerge, clinicians will be better positioned to make decisions about optimal placement of the various advanced therapies. In this context, "big data" and realworld evidence consortia have become pivotal in changing practice, especially for newer agents [16, 17, 22, 23].

Our analysis of the 50 policies included in Yadav et al. indicated that, among plans where coverage was known, $14,698,687$ lives were impacted by the lack of consistency in coverage between IV and SC ustekinumab (Supplementary Data Content 5). These lives would be covered for ustekinumab IV under medical benefits but not for ustekinumab $\mathrm{SC}$ under pharmacy benefits, rendering the IV induction/SC maintenance regimen recommended in the label impractical for clinical use. An additional 957,906 lives may also be impacted among those where coverage status of IV formulation is not known (Supplementary Data Content 5). This discordance between medical and pharmacy benefits presents an unnecessary hurdle in real-world practice and potentially compromises continuity of care for patients.

The strengths of our analysis include the large number of policies (medical: 3185; pharmacy: 6653) and covered lives $(301,748,186$ [medical benefit] and 302,057,543 [pharmacy benefit]) reviewed, real-time assessment of insurance coverage using the MMIT database, and ability to compare coverage patterns between biologics and across regions. This study is, however, subject to limitations. Data for this study were extracted from the MMIT database, and therefore our work was based on analyzing insurance policy coverage and is subject to the limitations of the policies we reviewed. We did not complement these analyses with any survey data and did not inquire about the need for a step-up requirement for coverage, which could have provided a richer real-world perspective on access, a valuable point to consider in further studies. Furthermore, access was assessed for the indication of $\mathrm{UC}$ and $\mathrm{CD}$, and the diagnosis was not confirmed.

In conclusion, contrary to previously published data, this analysis found that approximately half of covered lives have access to all biological agents (except tofacitinib) as firstline therapy for UC and CD and two-thirds had access after one biologic exposure. IBD management has traditionally been divided into 1 mechanism of action and two routes of administration. This dichotomy has worked to funnel patients into a therapy based on route of administration and coverage instead of value and risk-based assessment. With the emergence of new therapies, including biosimilars, there will be an increased pressure on payers to manage therapies and an increased responsibility on physicians to choose the most appropriate agent. It is important that modern physicians understand how to navigate coverage policies and advocate to create an individualized treatment pathway for each patient.

Acknowledgments The authors would like to thank Rachel Twardowski, MS, and Lisa Gramarossa, employees of Takeda 
Pharmaceuticals USA, Inc., for their contribution to the analyses and data interpretation. Medical writing assistance was provided by Reem Berro, PhD, and Anna Kaufman, MPH, of Syneos Health and supported by Takeda Pharmaceuticals USA, Inc.

Author's contribution All authors were involved in drafting the manuscript and critical revision of the manuscript for important intellectual content, and all authors approved the final version of the manuscript.

Funding This study was supported by Takeda Pharmaceuticals USA, Inc.

\section{Compliance with Ethical Standards}

Conflict of interest MTO reports: research grant from UCB; consulting fees from AbbVie, Janssen, Lycera, Merck, Pfizer, Takeda, UCB. WJS reports: research grants from Atlantic Healthcare Limited, Amgen, Genentech, Gilead Sciences, AbbVie, Janssen, Takeda, Lilly, Celgene/Receptos; consulting fees from AbbVie, Allergan, Amgen, Boehringer Ingelheim, Celgene, Conatus, Cosmo, Escalier Biosciences, Ferring, Genentech, Gilead, Janssen, Lilly, Miraca Life Sciences, Nivalis Therapeutics, Novartis, Nutrition Science Partners, Oppilan Pharma, Otsuka, Paul Hastings, Pfizer, Precision IBD, Progenity, Prometheus Laboratories, Ritter Pharmaceuticals, Robarts Clinical Trials, Salix, Shire, Seres Therapeutics, Sigmoid Biotechnologies, Takeda, TiGenix, Tillotts Pharma, UCB, Vivelix; and stock options from Ritter Pharmaceuticals, Oppilan Pharma, Escalier Biosciences, Precision IBD, Progenity. PSD reports: research support from Takeda and Pfizer; consulting and travel support from Takeda. CC, FR, and KL are employees of Takeda Pharmaceuticals USA.

Open Access This article is distributed under the terms of the Creative Commons Attribution-NonCommercial 4.0 International License (http://creativecommons.org/licenses/by-nc/4.0/), which permits any noncommercial use, distribution, and reproduction in any medium, provided you give appropriate credit to the original author(s) and the source, provide a link to the Creative Commons license, and indicate if changes were made.

\section{References}

1. Lichtenstein GR, Abreu MT, Cohen R, Tremaine W. American Gastroenterological Association. American Gastroenterological Association Institute technical review on corticosteroids, immunomodulators, and infliximab in inflammatory bowel disease. Gastroenterology. 2006;130:940-987.

2. Ungaro R, Mehandru S, Allen PB, Peyrin-Biroulet L, Colombel JF. Ulcerative colitis. Lancet. 2017;389:1756-1770.

3. Torres J, Mehandru S, Colombel JF, Peyrin-Biroulet L. Crohn's disease. Lancet. 2017;389:1741-1755.

4. Panaccione R, Rutgeerts P, Sandborn WJ, Feagan B, Schreiber $\mathrm{S}$, Ghosh S. Review article: treatment algorithms to maximize remission and minimize corticosteroid dependence in patients with inflammatory bowel disease. Aliment Pharmacol Ther. 2008;28:674-688.

5. Colombel JF, Narula N, Peyrin-Biroulet L. Management strategies to improve outcomes of patients with inflammatory bowel diseases. Gastroenterology. 2017;152:351-361.
6. Dassopoulos T, Cohen RD, Scherl EJ, Schwartz RM, Kosinski L, Regueiro MD. Ulcerative colitis care pathway. Gastroenterology. 2015;149:238-245.

7. Sandborn WJ. Crohn's disease evaluation and treatment: clinical decision tool. Gastroenterology. 2014;147:702-705.

8. Rubin DT, Uluscu O, Sederman R. Response to biologic therapy in Crohn's disease is improved with early treatment: an analysis of health claims data. Inflamm Bowel Dis. 2012;18:2225-2231.

9. Yadav A, Foromera J, Feuerstein I, Falchuk KR, Feuerstein JD. Variations in health insurance policies regarding biologic therapy use in inflammatory bowel disease. Inflamm Bowel Dis. 2017;23:853-857.

10. Entyvio [package insert]. Deerfield, IL: Takeda Pharmaceuticals America, Inc; 2018.

11. Wyant T, Fedyk E, Abhyankar B. An overview of the mechanism of action of the monoclonal antibody vedolizumab. J Crohns Colitis. 2016;10:1437-1444.

12. Stelara [package insert]. Horsham, PA: Janssen Biotech, Inc; 2018.

13. Xeljanz [package insert]. New York, NY: Pfizer Inc; 2018.

14. Colombel JF, Sands BE, Rutgeerts P, et al. The safety of vedolizumab for ulcerative colitis and Crohn's disease. Gut. 2017;66:839-851.

15. Lasch K, Liu S, Ursos L, et al. Gastroenterologists' perceptions regarding ulcerative colitis and its management: results from a large-scale survey. Adv Ther. 2016;33:1715-1727.

16. Narula N, Peerani F, Meserve J, et al. Vedolizumab for ulcerative colitis in clinical practice: treatment outcomes from the VICTORY consortium. Am J Gastroenterol. 2018;113:1345-1354.

17. Dulai PS, Singh S, Jiang X, et al. The real-world effectiveness and safety of vedolizumab for moderate-severe Crohn's disease: results from the US VICTORY consortium. Am J Gastroenterol. 2016;111:1147-1155.

18. Singh S, Fumery M, Sandborn WJ, Murad MH. Systematic review with network meta-analysis: first- and second-line pharmacotherapy for moderate-severe ulcerative colitis. Aliment Pharmacol Ther. 2018;47:162-175.

19. D'Haens G, Baert F, van Assche G, et al. Early combined immunosuppression or conventional management in patients with newly diagnosed Crohn's disease: an open randomised trial. Lancet. 2008;371:660-667.

20. Colombel JF, Panaccione R, Bossuyt P, et al. Effect of tight control management on Crohn's disease (CALM): a multicentre, randomised, controlled phase 3 trial. Lancet. 2018;390:2779-2789.

21. Schreiber S, Reinisch W, Colombel JF, et al. Subgroup analysis of the placebo-controlled CHARM trial: increased remission rates through 3 years for adalimumab-treated patients with early Crohn's disease. J Crohns Colitis. 2013;7:213-221.

22. Icht $\mathrm{O}$, Yanai $\mathrm{H}$, Ron $\mathrm{Y}$, et al. Comparative study of two cohorts of newly diagnosed Crohn's disease demonstrates change in therapeutic strategy. Digestion. 2017;96:135-141.

23. Fiorino G, Manetti N, Armuzzi A, et al. The PROSIT-BIO cohort: a prospective observational study of patients with inflammatory bowel disease treated with infliximab biosimilar. Inflamm Bowel Dis. 2017;23:233-243.

Publisher's Note Springer Nature remains neutral with regard to jurisdictional claims in published maps and institutional affiliations. 